FSU-SCRI-97-112

\title{
A new method to measure the chiral condensate in lattice QCD using Wilson fermions
}

\author{
Khalil M. Bitar \\ SCRI, The Florida State University, \\ Tallahassee, FL 32306-4130, USA \\ and \\ American University of Beirut, Lebanon \\ Urs M. Heller and Rajamani Narayanan \\ SCRI, The Florida State University, \\ Tallahassee, FL 32306-4130, USA
}

November 13, 2017

\begin{abstract}
Parity and flavor symmetry is not broken in QCD. Using this fact, we propose a new method to measure the chiral condensate in lattice QCD using Wilson fermions with an operator that breaks parity and flavor in addition to the chiral symmetry.
\end{abstract}

PACS \#: 12.38.-t, 11.30.Rd, 11.15.Ha.

Key Words: QCD, Chiral symmetry, Wilson fermions.

\section{Introduction}

Formal arguments in the continuum have shown that parity and flavor symmetries are not broken in QCD [1, 2]. On the other hand chiral symmetry is expected to be spontaneously broken in massless QCD with pions being the associated Goldstone bosons. A non-vanishing density of eigenvalues around 
zero for the massless Dirac operator is responsible for the breaking of the chiral symmetry[3]. A promising way to test the above statements in a rigorous non-perturbative setting is the lattice formulation of QCD. Attempts have been made to study these issues in lattice QCD using Wilson fermions and staggered fermions. For Wilson fermions chiral symmetry is broken explicitly by the Wilson term needed to remove the unwanted doublers on the lattice. This makes it difficult to study the spontaneous breaking of chiral symmetry using Wilson fermions since there is an additive renormalization to the bare fermion bilinear used to measure the chiral condensate on the lattice. Staggered fermions do realize massless fermions on the lattice but the continuum notion of chirality is not well defined for staggered fermions due to their inherent lattice construction.

Although parity and flavor symmetry is not broken in the continuum theory there are claims that it could be broken on the lattice in the context of Wilson fermions [1]. If this statement extends to the continuum limit, then it is not clear if Wilson fermions do realize QCD. A recent numerical investigation has provided some evidence that parity and flavor symmetry is probably not broken by Wilson fermions as one approaches the continuum limit [5]. In this letter we show that parity and flavor symmetry is not broken by Wilson fermions on the lattice even away from the continuum limit. As a consequence we propose a parity-flavor breaking operator as a good way to probe the spontaneous breaking of chiral symmetry using Wilson fermions on the lattice.

We first discuss the issue of parity and flavor symmetry in the continuum. We emphasize that the result for the preservation of flavor symmetry in [2] is only valid for the massive theory although it can be extended to the massless case with some care. As for parity, we note that one can rotate a $m \bar{\psi} \psi$ term into an $i h \bar{\psi} \gamma_{5} \psi$ term by a chiral rotation. One has to take the limit $h \rightarrow 0$ for a finite $m$ to study the violation of parity in massive QCD. On the other hand, one can approach the $m=h=0$ limit in the complex $(m, h)$ plane in any direction to measure the chiral condensate. With the above continuum statements in place, we focus on the lattice formulation of two flavor QCD using Wilson fermions. By introducing a parity and flavor breaking term, we show that parity and flavor symmetry is not broken in the massive theory realized using Wilson fermions. In the massless theory, statements similar to the continuum hold and this leads us to probe the chiral condenstate using an operator that breaks parity and flavor symmetry with no need for 
subtractions.

\section{Continuum}

The fermionic part of the continuum Euclidean action for QCD with explicit parity, flavor and chiral breaking terms is

$$
S=\sum_{f}\left[\bar{\psi}_{f} \not D \psi_{f}+m_{f} \bar{\psi}_{f} \psi_{f}+i h_{f} \bar{\psi}_{f} \gamma_{5} \psi_{f}\right]
$$

The subscript $f$ stands for the flavor index and $\not D=\gamma_{\mu} D_{\mu}$ is the usual Euclidean massless Dirac operator in a background gauge field . $D_{\mu}(A)$, $\mu=1,2,3,4$ are the four covariant derivative operators and they are antiHermitean. All the $\gamma_{\mu}$ 's are Hermitean and therefore $\not D$ is an anti-Hermitean operator. Note that $\bar{\psi}_{f}$ and $\psi_{f}$ are independent degrees of freedom in Euclidean space. We recall that $\left\{\gamma_{5}, \not D\right\}=0$ and that $\gamma_{5} \not D$ is a Hermitean operator. Under a chiral rotation given by

$$
\psi_{f} \rightarrow e^{i \theta \gamma_{5}} \psi_{f} ; \quad \bar{\psi}_{f} \rightarrow \bar{\psi}_{f} e^{i \theta \gamma_{5}},
$$

Eqn.(11) goes into itself with

$$
\left(m_{f}, h_{f}\right) \rightarrow\left(m_{f} \cos (2 \theta)-h_{f} \sin (2 \theta), h_{f} \cos (2 \theta)+m_{f} \sin (2 \theta)\right)
$$

for each of the flavors. On the other hand, under a parity transformation, $\left(m_{f}, h_{f}\right) \rightarrow\left(m_{f},-h_{f}\right)$. A parity tranformation can be realized as a chiral rotation in the massless case and therefore some care has to be taken in dealing with the massless theory. We assume that the theory is formulated in a periodic box of finite volume and we will then take the limit of the volume going to infinity in order to study spontaneous breaking of symmetries. The spectrum is discrete in a finite volume and is given by

$$
\not D e_{n}=i \lambda_{n} e_{n} ; \quad \not D \gamma_{5} e_{n}=-i \lambda_{n} \gamma_{5} e_{n} ; \quad \lambda_{n}>0
$$

and the eigenfunctions form a complete orthnormal set, i.e,

$$
e_{n}^{\dagger} e_{m}=\delta_{m n} ; \quad e_{n}^{\dagger} \gamma_{5} e_{m}=0
$$


We can expand $\psi_{f}$ and $\bar{\psi}_{f}$ in this orthonormal basis as follows:

$$
\psi_{f}=\sum_{n}\left(\phi_{f}^{n} e_{n}+\chi_{f}^{n} \gamma_{5} e_{n}\right) ; \quad \bar{\psi}_{f}=\sum_{n}\left(\bar{\phi}_{f}^{n} e_{n}^{\dagger}+\bar{\chi}_{f}^{n} e_{n}^{\dagger} \gamma_{5}\right)
$$

where $\phi_{f}^{n}, \chi_{f}^{n}, \bar{\phi}_{f}^{n}$ and $\bar{\chi}_{f}^{n}$ are the new Grassmann variables in the eigenfunction basis and the change of basis has a Jacobian equal to unity. In this basis, the action decouples into various sectors with each sector associated with $e_{n}, \gamma_{5} e_{n}$. Eqn.(1) can be rewritten as

$$
S=\sum_{f} \sum_{n}\left[i \lambda_{n} \bar{\phi}_{f}^{n} \phi_{f}^{n}-i \lambda_{n} \bar{\chi}_{f}^{n} \chi_{f}^{n}+m_{f}\left(\bar{\phi}_{f}^{n} \phi_{f}^{n}+\bar{\chi}_{f}^{n} \chi_{f}^{n}\right)+i h_{f}\left(\bar{\phi}_{f}^{n} \chi_{f}^{n}+\bar{\chi}_{f}^{n} \phi_{f}^{n}\right)\right] .
$$

With this in place, one can write expressions for the two fermionic expectation values of interest in each flavor sector, namely,

$$
\begin{gathered}
\left\langle\bar{\psi}_{f} \psi_{f}\right\rangle_{A}=\sum_{n} \frac{2 m_{f}}{\lambda_{n}^{2}+m_{f}^{2}+h_{f}^{2}}, \\
i\left\langle\bar{\psi}_{f} \gamma_{5} \psi_{f}\right\rangle_{A}=\sum_{n} \frac{2 h_{f}}{\lambda_{n}^{2}+m_{f}^{2}+h_{f}^{2}},
\end{gathered}
$$

where both of the expectation values above are in a fixed gauge field background. $m_{f}^{2}+h_{f}^{2}$ is invariant under the chiral rotation given by Eqn.(3) and only this combination appears in the denominator of the above two equations. In the infinite volume limit, the sums in Eqns. (8) and (9) are replaced by the integrals,

$$
\begin{aligned}
\left\langle\bar{\psi}_{f} \psi_{f}\right\rangle_{A} & =\frac{m_{f}}{\sqrt{m_{f}^{2}+h_{f}^{2}}} \int_{-\infty}^{\infty} d \lambda \rho(\lambda) \frac{1}{\sqrt{\left(m_{f}^{2}+h_{f}^{2}\right)}+i \lambda}, \\
i\left\langle\bar{\psi}_{f} \gamma_{5} \psi_{f}\right\rangle_{A} & =\frac{h_{f}}{\sqrt{m_{f}^{2}+h_{f}^{2}}} \int_{-\infty}^{\infty} d \lambda \rho(\lambda) \frac{1}{\sqrt{\left(m_{f}^{2}+h_{f}^{2}\right)}+i \lambda},
\end{aligned}
$$

with $\rho(\lambda) d \lambda$ being the density of eigenvalues at $\lambda$. From Eqn.(蛋) it follows that $\rho(\lambda)$ is an even function of $\lambda$.

Vafa and Witten [2] showed that flavor symmetry is not broken in massive QCD by first noting that in the limit of infinite volume

$$
\left\langle\bar{\psi}_{1} \psi_{1}-\bar{\psi}_{2} \psi_{2}\right\rangle_{A}=\int_{-\infty}^{\infty} d \lambda \rho(\lambda)\left(\frac{1}{m_{1}+i \lambda}-\frac{1}{m_{2}+i \lambda}\right) .
$$


We have set $h_{1}=h_{2}=0$ in Eqn.(10) so that parity is not explicitly broken. Since $m_{1} \neq 0$ and $m_{2} \neq 0$ there is no singularity in the integration region and therefore each of the integrals separately is finite. In the limit of $m_{1}=m_{2}$ they will be the same and we will arrive at $\left\langle\bar{\psi}_{1} \psi_{1}-\bar{\psi}_{2} \psi_{2}\right\rangle_{A}=0$ for every gauge field background indicating that flavor symmetry is not broken in massive QCD.

A similar argument also shows that parity is not broken in massive QCD. Since $m_{f} \neq 0$, there is no singularity in the integration region of Eqn.(11) with $h_{f}=0$ and we have

$$
\left.\lim _{h_{f} \rightarrow 0} i\left\langle\bar{\psi}_{f} \gamma_{5} \psi_{f}\right\rangle_{A}\right|_{m_{f} \neq 0}=0
$$

This argument is different from the one presented in [1]. In [1], the authors make the assumption that the free energy is a smooth function of the parity breaking parameter $h$ and then show that parity cannot be broken by introducing an arbitrary parity breaking operator. It is not apriori clear if the assumption of the smoothness of the free energy as a function of $h$ is a valid one. A spontaneous breaking of parity could result in a discontinuity in the slope of the free energy at $h=0$. Here we have considered a fermionic operator that breaks parity and have shown that its expectation value is zero.

Care has to be taken in extending the arguments above to massless QCD. Following [3] we note that

$$
\lim _{\mu \rightarrow 0} \int d \lambda \rho(\lambda) \frac{i}{\lambda \pm i \mu}= \pm \pi \rho(0)+i \int d \lambda \rho(\lambda) P\left(\frac{1}{\lambda}\right)
$$

where $P\left(\frac{1}{\lambda}\right)$ denotes the principle value of $\frac{1}{\lambda}$ and is an odd function of $\lambda$. The first term arises from the singularity at $\lambda=0$ in the limit of $\mu=0$. If we set $m_{f}=m$ and $h_{f}=h$, we have

$$
\left.\lim _{h \rightarrow 0^{+}} i\left\langle\bar{\psi} \gamma_{5} \psi\right\rangle_{A}\right|_{m=0}=\left.\lim _{m \rightarrow 0^{+}}\langle\bar{\psi} \psi\rangle_{A}\right|_{h=0}=\pi \rho(0)
$$

where we have used Eqn. (14) with $\mu=m$ and $\mu=h$. If $\rho(0) \neq 0$ we have a spontaneous breakdown of chiral symmetry. In fact one can approach the chiral limit $(m=h=0)$ along any direction in the complex $(m, h)$ plane and measure the magnitude of the chiral condensate.

To study flavor symmetry breaking in massless QCD, we proceed as follows. We set $h_{f}=0$ so that the only term that breaks flavor symmetry are 
the mass terms. In the massless limit,

$$
\lim _{m_{f} \rightarrow 0}\left\langle\left.\left(\bar{\psi}_{f} \psi_{f}\right\rangle_{A}\right|_{h=0}=\pi \frac{m_{f}}{\left|m_{f}\right|} \rho(0)\right.
$$

and this will be non-zero since chiral symmetry is expected to be broken in the massless limit. Clearly,

$$
\lim _{m_{1} \rightarrow 0} \mid\left\langle\left.\left(\bar{\psi}_{1} \psi_{1}\right\rangle_{A}\right|_{h=0}\left|=\lim _{m_{2} \rightarrow 0}\right|\left\langle\left.\left(\bar{\psi}_{2} \psi_{2}\right\rangle_{A}\right|_{h=0}\right|\right.
$$

and therefore the flavor symmetry is not broken. Note that

$$
\lim _{m_{1} \rightarrow 0^{+}}\left\langle\left.\left(\bar{\psi}_{1} \psi_{1}\right\rangle_{A}\right|_{h=0}=-\lim _{m_{2} \rightarrow 0^{-}}\left\langle\left.\left(\bar{\psi}_{2} \psi_{2}\right\rangle_{A}\right|_{h=0}\right.\right.
$$

But this is just another way to see the breaking of chiral symmetry. A similar statement holds if we study flavor symmetry breaking by setting $m_{f}=0$ and approach $h_{f} \rightarrow 0$.

We close this section by remarking that the eigenvalues of $\mathrm{H}(m)=\gamma_{5}(\not D+$ $m)$ are $\pm \sqrt{\lambda_{n}^{2}+m^{2}}$ with the same $\lambda_{n}$ 's as in Eqn.(四). The spectrum is real and has a gap when $m \neq 0$. It is natural to consider $\mathrm{H}(m)$ in the context of Wilson fermions on the lattice.

\section{Lattice}

We consider the issue of spontaneous breaking of chiral, parity and flavor symmetry on the lattice in the context of Wilson fermions. The lattice equivalent of $\mathrm{H}(m)$ is

$$
\mathrm{H}_{L}(m)=\left(\begin{array}{cc}
\mathrm{B}-m & \not \subset \\
\not^{\dagger} & -\mathrm{B}+m
\end{array}\right)
$$

with $\not=\sum_{\mu} \sigma_{\mu} C_{\mu}$ and $\mathrm{B}=\sum_{\mu} \mathrm{B}_{\mu} . \sigma_{1}, \sigma_{2}, \sigma_{3}$ are the usual Pauli matrices and $\sigma_{4}=i I . \quad C_{\mu}$ and $\mathrm{B}_{\mu}$ are the symmetric first covariant derivative and second covariant derivative on the lattice respectively. $m$ is the bare lattice mass. The B part of $\mathrm{H}_{L}(m)$ is $\gamma_{5}$ times the usual Wilson term. The lattice action is

$$
\sum_{f} \psi_{f}^{\prime} H_{L}(m) \psi_{f}+i h_{f} \psi_{f}^{\prime} \psi_{f}
$$


$\psi_{f}^{\prime}$ is related to the conventional $\bar{\psi}_{f}$ by $\bar{\psi}_{f}=\psi_{f}^{\prime} \gamma_{5}$. Following [4], 5], we have introduced a parity breaking term equal to $i h_{f} \psi_{f}^{\prime} \psi_{f}$. Since both flavors couple to the same gauge field and have the same lattice mass, the only flavor symmetry breaking term arises from the different couplings to the parity breaking term. In particular we will consider two flavor QCD with $h_{1}=-h_{2}=h$ so that we have one coupling that breaks both parity and flavor symmetry. We can then study the theory in the limit of $h \rightarrow 0$. In addtion to $h$ we have the lattice gauge coupling, $\beta$ and the lattice mass $m$. The functional integral is positive definite for all values of $m, h$ and $\beta$. The gauge action is positive as always and the result of the fermion integral is $\operatorname{det}\left(\mathrm{H}_{L}^{2}+h^{2}\right)$ which is positive definite.

Like in the continuum, the expectation value of the parity-flavor breaking term in a fixed gauge field background on an infinite lattice is

$$
\left\langle i\left(\bar{\psi}_{1} \gamma_{5} \psi_{1}-\bar{\psi}_{2} \gamma_{5} \psi_{2}\right)\right\rangle_{A}=\int_{-\infty}^{\infty} d \lambda \rho(\lambda)\left[\frac{i}{\lambda+i h}-\frac{i}{\lambda-i h}\right] .
$$

Here the $\lambda$ 's are the eigenvalues of $\mathrm{H}_{L}(m)$ on the lattice. The eigenvalues can be positive and negative but are bounded. In the continuum limit $\rho(\lambda)$ will be an even function of $\lambda$ but this will not be the case for finite $\beta$. If the spectrum has a gap, the integral is well defined and finite and therefore $\lim _{h \rightarrow 0}\left\langle i\left(\bar{\psi}_{1} \gamma_{5} \psi_{1}-\bar{\psi}_{2} \gamma_{5} \psi_{2}\right)\right\rangle_{A}=0$ and there is no breaking of parity or flavor symmetry. This is indeed the case if $m<m_{c}(\beta)$ where the lattice theory describes massive fermions. If the spectrum does not have a gap, then inserting Eqn.(14) into Eqn.(21) yields

$$
\lim _{h \rightarrow 0}\left\langle i\left(\bar{\psi}_{1} \gamma_{5} \psi_{1}-\bar{\psi}_{2} \gamma_{5} \psi_{2}\right)\right\rangle_{A}=2 \pi \rho(0)
$$

This shows that a non-zero result for the parity-flavor breaking operator on an infinite lattice at any finite value of $\beta$ arises from a non-vanishing spectral density of $\mathrm{H}_{L}(m)$ at zero. As remarked in the continuum analysis this is simply a signal for the spontaneous breakdown of chiral symmetry. In particular if we had set the parity breaking term for both flavors to be of the same sign and then considered Eqn.(21), we would have found that the $\rho(0)$ contribution from the two flavors cancel and we would have obtained a zero result indicating that flavor symmetry is not broken just like in the massive theory.

In the continuum limit, we expect the spectrum to have a gap if $m \neq 0$ and the gap to close at $m=0$. At a finite $\beta$ the B term in Eqn.(19) plays a 
role and the effective mass of the fermion is gauge field dependent. As such there cannot be a precise value of $m$ where the gap closes. This is evident when one studies topological issues using $\mathrm{H}_{L}(m)$ [6]. For a topologically non-trivial configuration on the lattice, $\mathrm{H}_{L}(m)$ must have a zero eigenvalue for some value of $m$ [7]. The value of $m$ where one gets a zero eigenvalue is a function of the size of the topological object. If we take the point of view that a non-zero $\rho(0)$ is a consequence of the gauge field configuration containing instantons and anti-instantons, we expect $\rho(0)$ to be non-zero for a range of $m$ if the lattice coupling is finite. Even if we do not accept the above point of view it is likely that there is a region of $m$ where $\rho(0)$ is nonzero since there is no precise definition of a massless point at a finite $\beta$. For Wilson fermions, the region of $m$ where $\rho(0)$ is non-zero is of order of the lattice spacing and will thus shrink to a point at $m=0$ in the continuum limit. $\lim _{h \rightarrow 0}\left\langle i\left(\bar{\psi}_{1} \gamma_{5} \psi_{1}-\bar{\psi}_{2} \gamma_{5} \psi_{2}\right)\right\rangle_{A}$ will be non-zero over a region of $m$ on the lattice at finite $\beta$. In this whole region of $m$ we are simply measuring a breakdown of chiral symmetry and we could say that a measure of the total chiral symmetry on the lattice is to sum the condensate over the whole region of $m$. This argument leads us to the following proposal for the measurement of the chiral condensate using Wilson fermions on the lattice:

$$
<\bar{\psi} \psi>=\frac{1}{2} \lim _{\beta \rightarrow \infty} \frac{1}{m_{2}(\beta)-m_{1}(\beta)} \int_{m_{1}(\beta)}^{m_{2}(\beta)} d m \lim _{h \rightarrow 0} \lim _{V \rightarrow \infty}\left\langle i\left(\bar{\psi}_{1} \gamma_{5} \psi_{1}-\bar{\psi}_{2} \gamma_{5} \psi_{2}\right)\right\rangle
$$

The order of the limits are standard. We first take the infinite volume limit before taking the coefficient of the parity-flavor breaking term to zero. For finite $\beta$ we will get a non-zero result if $m_{1}(\beta) \leq m \leq m_{2}(\beta)$ justifying the integral over this region of $m$. The continuum limit is taken at the very end. In the continuum limit both $m_{1}(\beta)$ and $m_{2}(\beta)$ approach zero and the integral reduces to the value of the integrand at $m=0$. We note that the second term in Eqn.(14) contributes to $\left\langle i \bar{\psi}_{i} \gamma_{5} \psi_{i}\right\rangle_{A}$ in Eqn.(21) for a finite $\beta$ since $\rho(\lambda)$ is not an even function of $\lambda$. But this contribution is zero in the continuum limit and is exactly cancelled between the two terms in Eqn.(23) on the lattice. 


\section{Conclusions}

We have considered the issue of parity and flavor symmetry breaking in QCD. By an extension of the standard analysis in the continuum [3, 2] we have shown that parity and flavor symmetry is not broken in massless QCD. Similar arguments show that parity and flavor symmetry is not broken in the lattice formulation of QCD using Wilson fermions. This lead us to propose a measurement of the breakdown of chiral symmetry using an operator that breaks parity and flavor symmetry in lattice QCD with Wilson fermions. An advantage of this proposal is that the operator does not suffer from any additive renormalization. Like in any numerical study of a spontaneous breakdown of a symmetry, it should be possible to use results on several finite $V$ 's and several non-zero $h$ 's and estimate the limit as $V \rightarrow \infty$ and $h \rightarrow 0$ by standard extrapolations.

Acknowledgements: We would like to thank Robert Edwards and Ivan Horvath for useful discussions. This research was supported by DOE contracts DE-FG05-85ER250000 and DE-FG05-96ER40979.

\section{References}

[1] C. Vafa and E. Witten, Phys. Rev. Lett. 53 (1984) 535.

[2] C. Vafa and E. Witten, Nucl. Phys. B234 (1984) 173.

[3] T. Banks and A. Casher, Nucl. Phys. B169 (1980) 103.

[4] S. Aoki, Prog. Theor. Phys. 122 (1996) 179. For a recent review please see, A. Ukawa, Nucl. Phys. (Proc.Suppl.) 53 (1997) 106.

[5] K. Bitar, Phys. Rev. D56 (1997) 2736; hep-lat/9709086.

[6] R. Narayanan and P. Vranas, hep-lat/9702005, To appear in Nuclear Physics B; R. Narayanan and R. Singleton Jr., hep-lat/9709016.

[7] R. Narayanan and H. Neuberger, Phys. Rev. Lett. 71 (1993) 3251; Nucl. Phys. B443 (1995) 305. 